\title{
Soccer Scoring Techniques - a Scientific Re-conception of Time and Space via Biomechanical Modeling
}

\author{
Xiang Zhang ${ }^{1,+}$ and Gongbing Shan ${ }^{2,1, *,+}$ \\ 1 Department of Physical Education, Xinzhou Teachers' University, Xinzhou, Shanxi 034000, China; \\ xiangzhang@xztc.edu.cn \\ 2 Biomechanics Lab, Faculty of Arts \& Science, University of Lethbridge, Lethbridge, AB T1K 3M4, Canada \\ * Correspondence: g.shan@uleth.ca; Tel.: +1-403-329-2683 \\ + The authors contributed equally to this work.
}

\begin{abstract}
From a scientific standpoint, both temporal and spatial variables must be examined when developing programs for training various soccer scoring techniques (SSTs), but a review of current literature reveals that existing scientific studies have overlooked this combinatory influence. Consequently, there is no reliable theory on temporal-spatial identification when evaluating scoring opportunities. Quantified by using biomechanical modeling, anthropometry, and SSTs found in FIFA Puskás Award (121 nominated goals between 2009 and 2020), it is found that players' proprioceptive/effective shooting volume (i.e. players' attack space) could be sevenfold the currently-practiced shooting volume. The ignorance of some SSTs' training leads to the underuse of the potential shooting volume. These overlooked SSTs are airborne and/or acrobatic techniques, perceived as high-risk and low-reward. Relying on the talent of an athlete to improvise on the fly can hardly be considered as a viable coaching strategy. Therefore, for developing science-based SST training regimes, groundbreaking studies are needed to: 1) expand the perception of shooting volume, and 2) entrain one-touch-shot techniques (airborne/acrobatic) within this volume, in short, Focusing-on-Time-in-Space. Whence, the new temporal-spatial theory could guide future researches and develop novel training programs. An increase of airborne/acrobatic goals would ultimately further enhance the excitement of the game.
\end{abstract}

Keywords: scoring opportunity identification; proprioceptive shooting volume; 0 possession shot; airborne; anthropometry

\section{Introduction}

Soccer is the most popular sport in the world. It is played and watched on six continents with 265 millions of players and 4 billions of fans, i.e. over 50\% of world population ( 7.7 billions) are linked to soccer [1-3]. Unfortunately, the scientific understanding of this sport lags far behind its practice, with most participants acquiring soccer scoring techniques (SST) through individual experience rather than science-based instruction [4-6]. Especially, there is a dearth of scientific study on some complex scoring techniques such as jumping turning kick (e.g. a nominated goal of FIFA Puskás Award 2019 performed by Ibrahimović [7]) and Diving scorpion kick (the FIFA Puskás Award 2017 [8]) [9,10]. The novelty of these unusual SSTs performed by talented athletes could imply the necessity of scientific discovery. Thus skills that are virtuosic in appearance may be eminently trainable and less a product of improvisatory abilities of individual players.

From a scientific standpoint, both temporal and spatial variables must be examined when developing programs for training various SSTs. But, a search using keywords in the authority database-Web of Science-reveals the following scenario: When the keyword "soccer goal" is applied, 3357 articles are found. However, when the keyword is changed to "soccer goal 
opportunity", the article number is dramatically dropped to 142. Even more theatrical, when additional keyword "possession" (temporal factor) or "positioning" (spatial factor) is added for a search, the number decreased to 31 and 33, respectively. To the end of the search, zero article occurs when the keywords "possession" and "positioning" are substituted by the "airborne ball" (i.e. three-dimensional space, Table 1). The review of current literature reveals that existing scientific studies have, at least, overlooked the influence of three-dimensional space on goal chance quantification. Consequently, there would be no reliable theory on temporal-spatial identification when evaluating soccer scoring opportunities.

Table 1. The result of literature search in all databases of Web of Science on 29 September 2020.

\begin{tabular}{ccccc}
\hline $\begin{array}{c}\text { Soccer } \\
\text { Goal }\end{array}$ & $\begin{array}{c}\text { Soccer Goal \& } \\
\text { Opportunity }\end{array}$ & $\begin{array}{c}\text { Soccer Goal \& } \\
\text { Opportunity \& } \\
\text { Possession }\end{array}$ & $\begin{array}{c}\text { Soccer Goal \& } \\
\text { Opportunity \& } \\
\text { Positioning }\end{array}$ & $\begin{array}{c}\text { Soccer Goal \& } \\
\text { Opportunity \& } \\
\text { Airborne Ball }\end{array}$ \\
\hline 3357 & 142 & 31 & 33 & 0 \\
\hline
\end{tabular}

A recent study [10] reveals that there are 43 SSTs existed in current soccer games, 26 out of the 43 are air-attack techniques (i.e. airborne shot). The result, in combination with Table 1, would suggest that groundbreaking researches are needed in order to develop science-based SST training regimes. The current paper aims to lay a foundation for launching the groundbreaking studies through a re-conception of temporal and spatial factors related to soccer shooting and to identify elements that could be applied in entrainment of SSTs via biomechanical modeling.

\section{Materials and Methods}

\subsection{Scietific Essentials regarding SSTs' Exploration}

In soccer, shots lead to goals and goals win games. As such, it would seem to be a logical focus for both researchers and practitioners to explore all means that might increase the number and effectiveness of shots taken during a game. Compared to many other sports, goals are relatively infrequent in soccer (on average $<3$ goals/game in World Cup since 1960s [11]); many games are won with just a single goal. Most of the game-play is dedicated to setting up or defending against scoring opportunities. If an attacker gets a scoring opportunity, his/her chance to shoot the ball typically does not last very long. In these brief moments, a player needs to shoot the ball quickly (a temporal aspect of goal scoring) and accurately (a spatial feature of goal scoring). These two parameters govern SSTs and are ultimately the determinants that decide whether the team's effort is rewarded.

In the current paper, these determinants are quantitatively explored through a novel theoretical framework. The framework is based on 1) temporal parameters identified in the past studies [12-16], 2) new spatial variables obtained by video-based analysis of all 121 nominated goals of FIFA Puskás Award (2009-2020) [16], and 3) quantification of the effective shooting volume of an athlete via biomechanical modeling. This effective shooting volume depends on a player's proprioceptive abilities, which are developed through structured repetitive trainings [18-21]. Hence the volume can also be named as players' proprioceptive shooting volume. Its quantification would decide if a ball should be counted as a goal chance (i.e. the ball is within/passing through the volume) or not.

\subsection{Selection of Temporal and Spatial Factors}

Through examination of the existing studies [12-16] and videos of the 121 nominated goals of FIFA Puskás Award, the following parameters that could be applied in the identification of the scoring opportunities were selected:

The possession number of ball before shooting [12-14] was used for the temporal identification. Three categories were applied in the current paper: 0 possessions (i.e. one-touch-shot where a player shoots as a ball is passing by), 1 possession (i.e. setting the ball and then shooting) and the other ball control strategies combined (i.e. $2+$ possession maneuvers). 
Regarding the spatial analysis, previous studies focused only on field-geography [14-16] and neglected the influences of both the athletes' body orientation (e.g. facing [22] or back-facing [9] the goal) and the spatial position of the ball at the instant of shot. To make the latter aspect in details, the spatial position of the ball have horizontal and vertical components. Horizontally, using the goal and the player as positional references, the ball can be between them, beyond them or to the side of them. Vertically, the ball can be airborne or on the ground. In the current study, these new spatial parameters related to athletes' body orientation and the spatial position of the ball at the instant of shot were selected for our quantification.

Figure 1 shows the selected temporal and spatial parameters. Using these parameters, all the 121 Puskás videos were quantitatively analyzed. Descriptive statistics (i.e. pie chart) is applied to summarize these categorical data (i.e. percentage distribution) in order to reveal the characteristics of the selected parameters and their contributions to scoring goals at the top elite level.

\subsection{Quantification of the shooting/attack volume through biomechanical modeling}

3D full-body biomechanical modeling was used to estimate an athlete's attack volume (i.e. proprioceptive/effective shooting volume). Logically, the larger one's effective shooting volume, the more goal chances one will possess. Through the full-body biomechanical modeling, the current article would first quantify the dimensions of various SSTs, and then, establish a link between SST and goal chance quantification. Such a conceptual organization is missing in the current soccer research and practice.

\section{Results}

The existing studies [12-14] examined more than 600 professional and World Cup matches. The results have shown that scoring with 0 possession (i.e. one-touch-shot) amounts for $26 \%-40 \%$ of goals. The longer a player possesses the ball, the lower the scoring chance. Setting the ball and then shooting (1 possession) is the next most successful strategy. Combined, 0 and 1 possession shots account for over $50 \%$ of goals. All other ball control strategies combined (2+ possession maneuvers) have lower success rates. The temporal branch of Figure 1 based on the 121 FIFA Puskás Award nominated goals of $2009-2020$ shows even more pronounced results: $57.0 \%$ ( 0 possession), $71.9 \%$ ( 0 \& 1 possession), and $28.1 \%$ (2+ possessions), respectively.
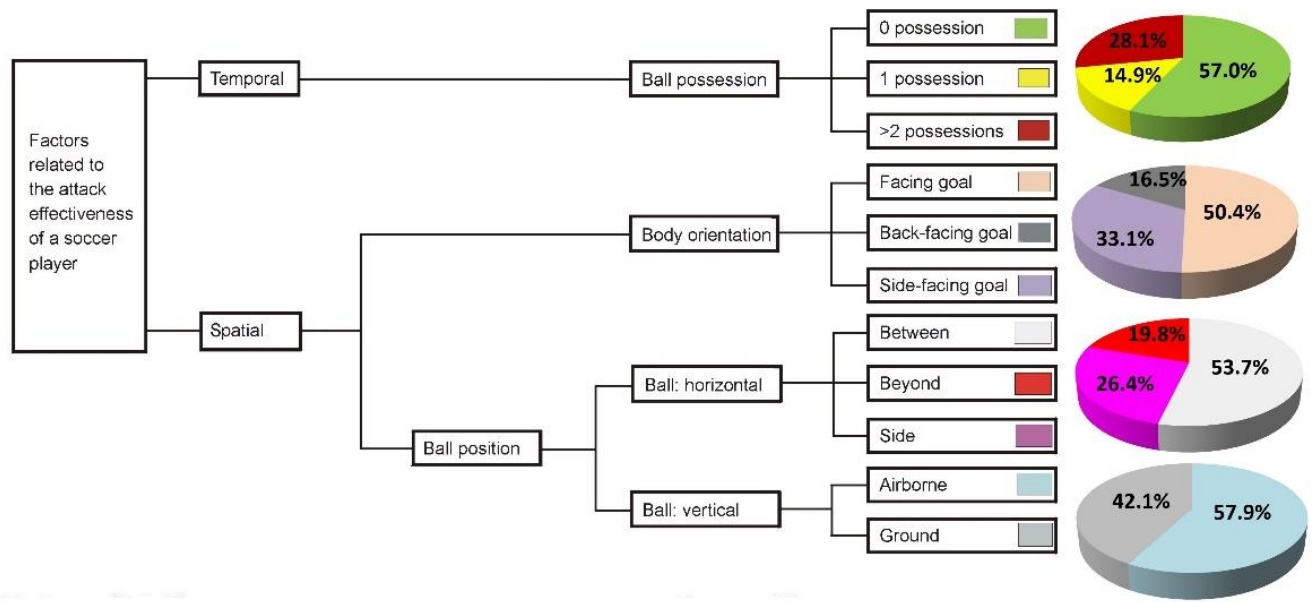

Figure 1. The temporal and spatial factors related to goal chance identification and their contributions to goals: empirical evidences drawn from all 121 FIFA Puskás Award nominees' goals 2009-2020.

The results of spatial analysis show that, as a ball comes into an athlete's proprioceptive shooting volume, a player's body orientation can be facing, side-facing, or back-facing (Figure 1 and Figure $2(\mathrm{~A})-(\mathrm{C}))$, relative to the position of the goal. 


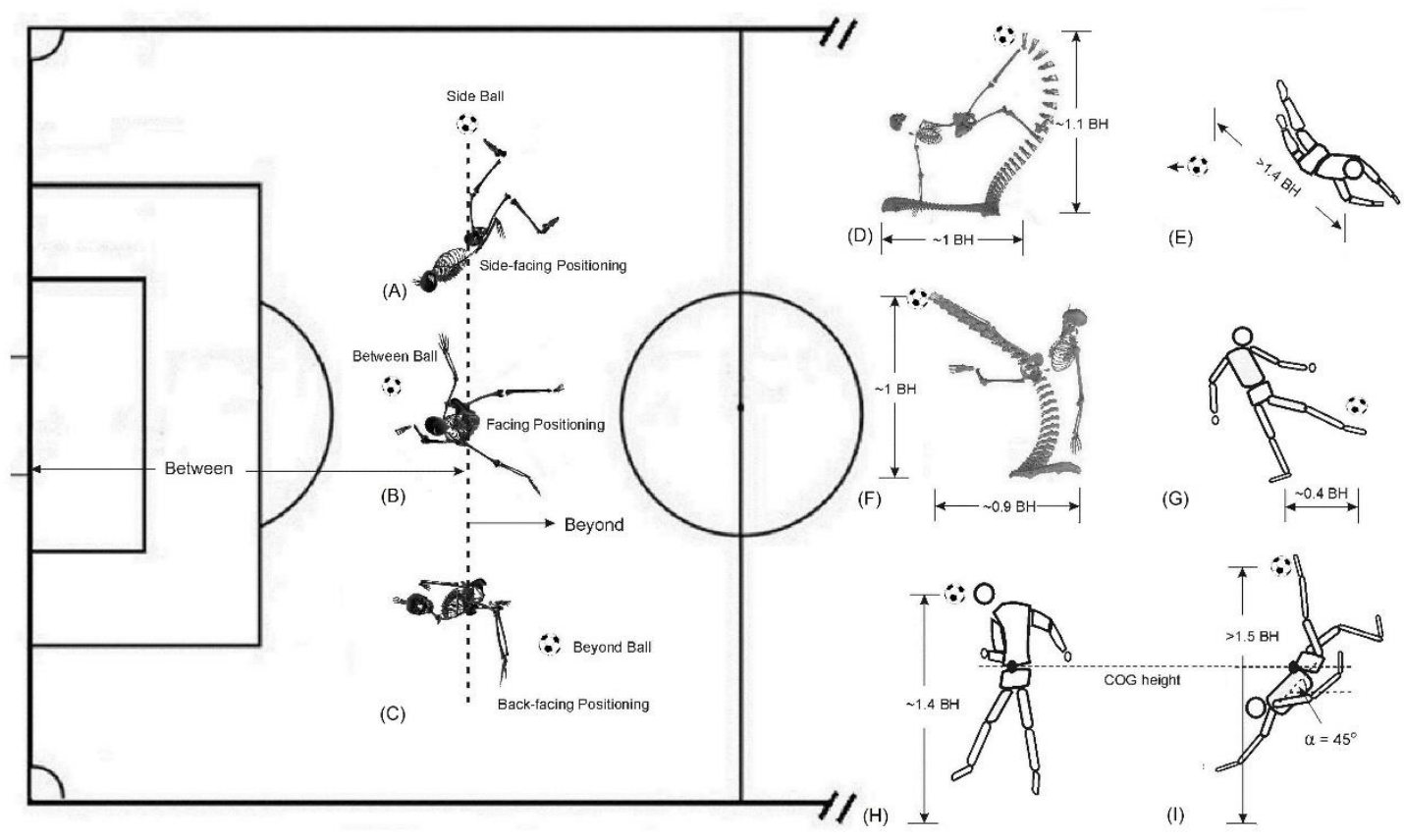

Figure 2. The clarification of spatial factors and their influences on the dimension quantification of the attack space (scoring chance): (A) 3D quantification of the jumping side volley (top-view) [23,24]. (B) 3D quantification of the maximal instep kick (top-view) $[25,26]$. (C) 3D quantification of the bicycle kick (top-view) [9,24]. (D) Dimensions of bicycle kick (side-view with timely trace of kick foot) obtained via 3D motion analysis and biomechanical modeling [9,24]. (E) Frontal dimension of a diving header - distance estimated using diving biomechanics [27] and anthropometrical modeling $[28,29]$. (F) Dimensions of the jumping side volley (frontal view with timely trace of kick foot) obtained via 3D motion analysis and biomechanical modeling [23,24]. (G) Lateral dimension of volley kick - distance estimated using biomechanical modeling and anthropometrical study [28]. (H) Vertical dimension of a jumping header - distance estimated using jump biomechanics [30,31] and anthropometrical modeling [28,29]. (I) potential attack height that might be reached using a bicycle kick at different trunk-angle orientations - biomechanical model estimation $[9,28]$.

In terms of body orientation at the moment of shooting, of the 121 Puskás goals, about half $(50.4 \%)$ were achieved facing the goal, while $33.1 \%$ were "side-facing", and $16.5 \%$ were "back-facing" (Figure 1). Horizontal ball position statistics are comparable, 53.7\% of goals occurred when the ball was between player and goal, while side and beyond balls accounted for the remaining 46.3\% (Figure 1). Regarding vertical ball positioning, 57.9\% of goals were airborne and only $42.1 \%$ were ground balls (Figure 1 ).

Table 2. A 1.8m-tall athlete's proprioceptive/effective shooting volume $\left(\mathrm{m}^{3}\right)$ quantified/estimated by using biomechanical modeling and anthropometrical data.

\begin{tabular}{ccc}
\hline $\begin{array}{c}\text { Currently } \\
\text { practiced }\end{array}$ & $\begin{array}{c}\text { Achieved by talented } \\
\text { elite athletes }\end{array}$ & $\begin{array}{c}\text { Theoretical } \\
\text { potential }\end{array}$ \\
\hline$\sim 8.5$ & $\sim 35.4$ & $\sim 58.8$ \\
\hline
\end{tabular}

The results of the volume quantification of the proprioceptive/effective shooting were shown in Table 2. In the current soccer coaching practice (i.e. the systematically trained SSTs are maximal instep kick, jumping header and volley/side volley [32,33]), the shooting volume formed by these skills in terms of a player's body height $(\mathrm{BH})$ as follows: anterior-posterior direction $-1.3 \mathrm{BH}$ (the last-stride-length of the maximal instep kick) [4,26], vertical direction - 1.4 BH (jumping height for a header) (Figure $2(\mathrm{H})$ ), and medial-lateral direction $-0.8 \mathrm{BH}$ (lateral reach of the side volley $-0.4 \mathrm{BH}$ each leg) (Figure $2(\mathrm{G})$ ). If we consider the case of a $1.8 \mathrm{~m}$ tall athlete, when the above is considered, 
the calculation of his/her maximum effective shooting volume would be $2.34 \mathrm{~m} \times 2.52 \mathrm{~m} \times 1.44 \mathrm{~m}$, or very roughly $8.5 \mathrm{~m}^{3}$. If the volumes of two acrobatic SSTs (i.e. bicycle kick and jumping side volley, Figure 2 (D) \& 2 (F) performed by some elite soccer players) are included in the calculation, the same player's effective shooting volume would be $4.14 \mathrm{~m} \times 2.52 \mathrm{~m} \times 3.24 \mathrm{~m}$, roughly $35.4 \mathrm{~m}^{3}$, or approximately four times the shooting volume of the normally practiced techniques in the current coaching practice. If additional SSTs such as the diving header (Figure 2 (E)) and an improved bicycle kick (trunk angled $45^{\circ}$ in addition to the current $0^{\circ}$ bicycle kick, Figure 2 (I)) are included, effective shooting volume increases to $4.32 \mathrm{~m} \times 2.70 \mathrm{~m} \times 5.04 \mathrm{~m}$, roughly $58.8 \mathrm{~m}^{3}$, or approximately seven times the normally practiced shooting volume.

\section{Discussion}

Due to the rarity of goal chance in soccer, it is highly relevant to establish a theoretical system to guide the research and coaching practice. Obviously, a scientific clarification/elaboration of a scoring chance and its relationship to the temporal and spatial concepts would form a foundation, on which scientific developments and improvements of SSTs could be built. Unfortunately, the scientific studies on the temporal-spatial influence on effectiveness of soccer attacks (i.e. turn a scoring chance into a goal) has not yet seen.

\subsection{Temporal-spatial Opportunity Identification}

In essence, scoring chances are an issue of maximizing probabilities of scoring, which cannot be determined without required basic theories/knowledge related to temporal-spatial identification and quantification of players' possibilities to shoot. It is important for researchers and practitioners to understand how the probability assessments could be made, and how they could contribute to goals.

Scoring opportunity can be defined by the feasibility of shooting successfully, i.e. a chance would be given for an attacker when a ball into a position where reaching the goal is both achievable and likely. Previous games' analyses have shown that such a scenario is an ephemeral "free ball" in or near penalty area $[12,14,16]$. These studies have shown that more than $65 \%$ of goals are scored when an attacking player possesses the ball in or near his/her opponents' penalty area, and he/she is not hindered by defenders - a "free ball". Hence, most goal-scoring studies (and training) overwhelmingly emphasize the field-geography of the ball $[13,14,16]$. As a consequence, the current practice and training programs [32,33] focus attention on team strategies to achieve geographic opportunity and underemphasizes temporal-spatial opportunity identification related to SSTs.

The evidences gained from the 121 FIFA Puskás goals (Figure 1) have indicated that a novel theoretical frame is needed to re-examine and/or allocate the known and unknown factors related to the existing SSTs and breakthrough studies are desired to quantify the influences of those factors on temporal-spatial opportunity identification.

\subsubsection{Temporal Efficiency}

The first challenge would be the temporal enumeration, i.e. the possession number of ball before shooting. Time is of the essence when preparing to shoot the ball. Even if in possession of a "free ball", a player will likely not be free for long; defenders will attempt to thwart the shot. The results summarized in this study clearly indicate that a sudden attack would create the highest temporal efficiency for converting a scoring chance into a goal. But, temporal aspect does not stand alone; obviously, it interacts with spatial factors. 


\subsubsection{Spatial Effectiveness}

Space is a more challenging concept; but it must certainly consist of more than mere field-geography. A player's proprioceptive abilities influence goal opportunity identification, i.e. as the soccer ball comes into a player's proprioceptive shooting volume, his/her ability of performing a shooting control under various body orientations and ball 3D spatial positions must fall within these abilities in order for him/her to attempt to score. Such proprioceptive abilities can be developed through repetitive trainings with structured/targeted training [18-21].

Based on the current literature there seems to be existing research and coaching emphasis on shots taken: 1) facing the goal, and 2) with the ball between the player and the goal $[32,33]$. It is not surprising that most training and practice regimes concentrate on the variables that account for the higher percentages of goals found in the pie charts of Figure 1. However, this neglects a significant percentage of scoring opportunities that may exist in smaller "slices of the pie". Some of these might be improved through structured training. For example, Swedish player Ibrahimovic's bicycle kick against England in 2013 (i.e. the Puskás award 2013) was a goal achieved as he ran away from, and not toward, the goal. This shot can be characterized as follows: 0 possession, back facing the goal, a beyond ball, and an air-attack. Currently, there is no science-based training program available for practitioners $[9,24]$. Therefore, the skill is generally considered as a product of the improvisatory and virtuosic abilities of an individual player, not as a trainable one. The question arises whether or not training regimes that develop SST shooting components required for such "virtuosity" might improve scoring by increasing players' proprioceptive/effective shooting volumes. The 121 Puskás goals have shown that $16.5 \%$ goals were achieved by using back-facing-goal techniques and $19.8 \%$ goals were with ball located beyond (not between) the goal and players (Figure 1). These "unusual" goals would signify that what is required is a theoretical framework to logically link the temporal and spatial factors for a systematical exploration.

\subsubsection{Lost in Time and Space in the Current Soccer Research}

From a scientific standpoint, both temporal and spatial variables must be examined when developing programs for SST training. However, our study reveals that the temporal-spatial aspect is mostly neglected in the current soccer research and coaching literature, i.e. scientific soccer studies are lost in time and space.

Further, the results of Puskás goals show that a few talented elite athletes, via long-time practice, have developed improvisatory abilities, which can turn "impossible" goals (commonly identified as non-chance goals) into goals. This study would suggest that scientific quantification would result in the scoring chance identification as "yes" or "no" instead of "impossible", if these skills could be demystified through a biomechanically quantitative study. Without a scientific quantification on temporal-spatial challenges, an identification of a scoring chance would be vague, subjective and unclear. Unfortunately, this is the situation in current soccer study, especially to the spatial challenges.

\subsection{Quantification of Athletes' Proprioceptive/Effective Shooting Volume - A Key for Scoring-opportunity Identification}

Currently, there are few, if any, studies on the manipulation/expansion of players' attack volume. Scientifically, such a volume is linked to a player's proprioceptive ability (i.e. athletes' proprioceptive/effective shooting volume) [18-21]. Previous studies on the proprioceptive ability have shown that such an ability is highly entrainable [19-21].

Discounting "lucky" goals, a player's goal scoring volume can be limited by the volume of those SSTs that athletes have been entrained. The normally entrained SSTs in the current coaching practice are: 1) maximal instep kick including the curved kick (characteristics: facing the goal, a between ball, \& a ground ball), 2) headers (characteristics: facing/side-facing the goal, a between/side ball, \& an air-attack), and 3) volley kick (characteristics: facing/side-facing the goal, a between/side ball, \& an air-attack below the hip) [32,33]. Therefore, the current training system would 
under-develop plyers' proprioceptive/effective shooting volume, reducing their possibilities to shoot/decreasing their goal chances.

The results of our study (Table 2) would suggest that the talent athletes (e.g. Cristiano Ronaldo and Zlatan Ibrahimović, the winners of Puskás award) have found ways (i.e. mastering unique acrobatic SSTs) to increase their proprioceptive/effective shooting volume by 4 times as compared to the normally trained players. These unusual SSTs contribute to enlarge their proprioceptive shooting volume. Further, our study has revealed that, theoretically, the increased volume could potentially further be enlarged by seven times of the "normally" practiced shooting volume (Table 2). The ramifications of our results are far reaching. Even if less than half of these theoretical gains could be realized through training of underutilized "slices of the pie", a player's effective proprioceptive shooting volume could be doubled or tripled.

Obviously, the dimensions of the proprioceptive shooting volume are heavily influenced by SSTs available to an athlete. A recent study [10] and the goals of Puskás nominees unveil that there are more than 10 SSTs that should be considered as potential contributors to maximize a player' proprioceptive shooting volume. Based on FIFA criteria ("Puskás goals should not be the result of luck, mistakes, deflection by another player or the player in an offside position") [17], these SSTs are clearly repeatable; so, they should be entrainable. What we need are scientific studies that demystify these SSTs and establish new and effective training regimes.

\subsection{Focusing on Time in Space: the new Framework/Nexus for Uniting Time Efficiency and Spatial Effectiveness}

The nexus for uniting the time efficiency and spatial effectiveness is the SSTs. First, mastering more SSTs would increase the effective dimension of the attack space to gain more scoring chances, i.e. the larger the effective dimension is, the more chances will be covered. Second, mastering more SSTs would ensure a shot with 0-ball possession regardless of ball's horizontal and vertical position and an attacker's dynamic posture (Figure 1 \& 2). Last, mastering more SSTs would enable an attacker to preclude a defense player for a clear shot, i.e. more options for choosing an attack.

Regrettably, scientific studies as well as coaching practice fall far behind the practice; most of the identified SSTs (currently 43 in total [10]) cannot be systematically trained due to the lack of scientific investigation and understanding $[9,23,24]$. The SSTs overlooked by researchers and coaches are the airborne and/or acrobatic attack-techniques such as scorpion kick, diving scorpion kick, jumping side volley, jumping turning kick, long-jump turning header, diving header, sliding kick and more [10]. The fatal effect of the overlook is the descent of the time efficiency and spatial effectiveness.

The common characteristics of the overlooked SSTs in research and training are exceedingly complex, labeled as high-risk and low award $[9,23,24]$. These skills, for most athletes, can hardly learn without insightful guidance. It is time for researchers to conduct quantitative studies for 1) demystifying the complexity of the skills; 2) identifying skills required for various ball positions and dynamic body postures; and 3) develop training programs for injury-free exercise, since the accurate performance of the airborne/acrobatic SSTs requires repetitive practice.

In short, merely relying on the talent of an athlete to improvise on the fly can hardly be considered a viable coaching strategy. A more structured conceptual organization of SSTs' training could improve players' spatial awareness in terms of body orientation and ball spatial position; applied practice of a one-touch-shot within this improved volume could concomitantly lead to increased scoring, i.e. Focusing-on-Time-in-Space would be the new framework and should act as the strategy for developing novel training programs.

\section{Conclusions}

Retrospectively, from a scientific standpoint, both temporal and spatial variables must be examined when evaluating soccer scoring opportunities. Unfortunately, field geography tends to dominate attention of researchers and practitioners, and consideration of player-centered temporal-spatial aspects of SSTs is mostly neglected, i.e. the research is Lost in Time and Space. 
Space certainly consists of more than mere field geography. A player's trained SSTs, i.e. his/her proprioceptive/effective shooting volume, influence both scoring opportunity identification and the dimensions of his/her attack space. The development of novel training programs should, first, focus on the increase of the attack space through mastering as many SSTs as possible; and then, concentrate on selecting a proper SST for reaching one-touch-shot in the attack volume, i.e. Focusing on Time in Space. The results of our biomechanical modeling reveals that an athlete has to learn airborne/acrobatic SSTs in order to increase his/her attack space. Therefore, scientific studies are needed to demystify the complexity of these skills for their learning and training.

The great attraction of soccer for millions of fans is the goal. Various techniques for gaining goals are sources of excitement. More frequent use of airborne/acrobatic SSTs for goals can only enhance the excitement of the game. Therefore, this new framework of Focusing on Time in Space would bring more excitement to us through promoting novel studies and developing innovative training programs for learning and practice various SSTs.

Author Contributions: X.Z. and G.S. designed the study, performed the data collection, analyzed and interpreted the data, prepared the draft, contributed to the revisions and proof reading of the article. All authors have read and agreed to the published version of the manuscript.

Funding: The research project was supported by Shanxi 100 Scholars' Grant (China) and National Sciences and Engineering Research Council of Canada (NSERC), grant number RGPIN-2014-03648.

Conflicts of Interest: The authors declare no conflict of interest. The founding sponsors had no role in the collection, analyses, or interpretation of data; in the writing of the manuscript, and in the decision to publish the results.

\section{References}

1. FIFA. 265 million playing football. 2007. Available online: https://www.fifa.com/mm/document/fifafacts/bcoffsurv/emaga_9384_10704.pdf (accessed on 16 June 2019).

2. Sawe, B. The Most Popular Sports In The World. 2018. Available online: https://www.worldatlas.com/articles/what-are-the-most-popular-sports-in-the-world.html (accessed on 16 June 2019).

3. Worldometers. Current World Population. 2018. Available online: http://www.worldometers.info/world-population/ (accessed on 16 June 2019).

4. Shan, G.; Westerhoff, P. Full-body kinematic characteristics of the maximal instep soccer kick by male soccer players and parameters related to kick quality. Sports Biomech. 2005, 4, 59-72.

5. Shan, G. Influences of Gender and Experience on the Maximal Instep Soccer Kick. Eur. J. Sport Sci. 2009, 9, 107-114.

6. Shan, G.; Zhang, X.; Wan, B.; Yu, D.; Wilde, B.; Visentin, P. Biomechanics of coaching maximal instep soccer kick for practitioners. Interdiscip. Sci. Rev. 2019, 44, 12-20.

7. FIFATV. FIFA PUSKAS AWARD 2019 NOMINEE: Zlatan Ibrahimovic. 2019. Available online: https://www.youtube.com/watch?v=oV6L2cNr0MU (accessed on 11 June 2020).

8. FIFATV. Olivier GIROUD - FIFA PUSKAS AWARD 2017 FINAL 3. 2019. Available online: https://www.youtube.com/watch?v=wGOhxt5Ksc0 (accessed on 11 June 2020).

9. Shan, G.; Visentin, P.; Zhang, X.; Hao, W.; Yu, D. Bicycle kick in soccer: Is the virtuosity systematically entrainable? Sci. Bull. 2015, 60, 819-821.

10. Zhang, X.; Shan, G.; Liu, F.; Yong, S.; Meng, M. Diversity of Scoring, Ingenuity of Striking, Art of Flying Conceptual and Systematical Identification of Soccer Scoring Techniques. Phys. Act. Rev. 2021, 9(1), 79-92.

11. FIFA. Average Number of Goals Scored per Game at the FIFA World Cup from 1930 to 2018. 2020. Available online: https://www.statista.com/statistics/269031/goals-scored-per-game-at-the-fifa-world-cup-since-1930/ (accessed on 26 January 2020).

12. Reep, C.; Benjamin, B. Skill and chance in association football. J. Royal Stat. Soc., 1968, A(131), 581-585.

13. Hughes, M.; Franks, I. Analysis of passing sequences, shots and goals in soccer. J. Sport. Sci. 2005, 23(5), 509-514. 
14. Wright, C.; Atkins,S.; Polman, R.; Jones, B.; Sargeson, L. Factors associated with goals and goal scoring opportunities in professional soccer. Int. J. Perform. Anal. Sport. 2011, 11(3), 438-449.

15. Bate, R. Football chance: tactics and strategy. In Science and football; Reilly, T., Lees, A., Davids, K., Murphy, W., Eds.; E. \& F. N. Spon Ltd: London, UK, 1988; pp. 293-301.

16. Seabra, F.; Dantas, L. Space definition for match analysis in soccer. Int. J. Perform. Anal. Sport. 2006, 6(2), 97-113.

17. FIFA. FIFA introduces new FIFA Puskás Award to honour the "goal of the year". 2009. Available online: https://www.fifa.com/mensyoutholympic/news/y=2009/m=10/news=fifa-introduces-new-fifa-puskas-awar d-honour-the-goal-the-year-1120531.html (accessed on 21 June 2019).

18. Sherrington, C. On the proprioceptive system, especially in its reflex aspect. Brain. 1907, 29, 467-482.

19. Wong, J.; Kistemaker, D.; Chin, A.; Gribble, P. Can proprioceptive training improve motor learning? J. Neurophysiol. 2012, 108(12), 3313-3321.

20. Alsmith, A.; Longo, M. Where exactly am I? Self location judgements distribute between head and torso. Conscious. Cogn. 2014, 24, 70-74.

21. Aman, J.; Elangovan, N.; Yeh, I.; Jürgen Konczak, J. The effectiveness of proprioceptive training for improving motor function: a systematic review. Front. Hum. Neurosci. 2015, 8, 1075.

22. Shan, G.; Zhang, X. From 2D Leg Kinematics to 3D Full-body Biomechanics - The Past, Present and Future of Scientific Analysis of Maximal Instep Kick in Soccer. Sports Med. Arthrosc. Rehabil. Ther. Technol. 2011, 3 , 23.

23. Zhang, X.; Shan, G.; Liu, F.; Yu, Y. Jumping Side Volley in Soccer - A Biomechanical Preliminary Study on the Flying Kick and Its Coaching Know-How for Practitioners. Appl. Sci. 2020, 10(14), 4785.

24. Shan, G. Biomechanical Know-how of Fascinating Soccer-kicking Skills-3D, Full-body Demystification of Maximal Instep Kick, Bicycle Kick \& Side Volley. In Proceedings of the 8th International Scientific Conference on Kinesiology, Zagreb, Opatija, Croatia, 10-14 May 2017.

25. Shan, G.; Daniels, D.; Wang, C.; Wutzke, C.; Lemire, G. Biomechanical analysis of maximal instep kick by female soccer players. J. Hum. Mov. Stud. 2005, 49, 149-168.

26. Shan, G.; Yuan, J.; Hao, W.; Gu, M.; Zhang, X. Regression equations for estimating the quality of maximal instep kick by males and females in soccer. Kinesiology 2012, 44, 139-147.

27. Blanksby, B.; Nicholson, L.; Elliott, B. Swimming: Biomechanical analysis of the grab, track and handle swimming starts: an intervention study. Sports Biomech. 2002, 1, 11-24.

28. Shan, G.; Bohn, C. Anthropometrical data and coefficients of regression related to gender and race. Appl. Ergon. 2003, 34, 327-337.

29. Zhang, X.; Shan, G.; Wang, Y.; Wan, B., Li, H. Wearables, biomechanical feedback, and human motor-skills' learning \& optimization. Appl. Sci. 2019, 9(2), 226.

30. Aragón, L. Evaluation of four vertical jump tests: Methodology, reliability, validity, and accuracy. Meas. Phys. Educ. Exerc. Sci. 2000, 4, 215-228.

31. Shergold, A. The height of perfection: Why Cristiano Ronaldo can jump higher than anyone else (MailOnline) 2013. Available online: https://www.dailymail.co.uk/sport/football/article-2278671/Cristiano-Ronaldo-Why-Real-Madrid-player-j ump-higher-else.html (accessed on 16 July 2018).

32. Thömmes, F. Fußballtraining für jeden Tag: die 365 besten Übungen; [+16 Seiten moderne Entwicklungstendenzen]. Stiebner Verlag GmbH: Grünwald, Germany, 2012.

33. FIFA. FIFA Coaching Manual. FIFA: Zurich, Swiss, 2017; pp. 160-260. 